\title{
Hepatocellular carcinoma with uncommon sites of metastasis: a rare case report
}

\author{
Mangala Targe ${ }^{1 *}$, Venkata Ramesh Yasam ${ }^{2}$ and Raj Nagarkar ${ }^{3}$
}

\begin{abstract}
Background: Hepatocellular carcinoma $(\mathrm{HCC})$ is a highly malignant tumor, causing both intrahepatic and extrahepatic metastases. The extrahepatic metastasis occurs in one-third of patients with HCC and it is associated with a poor prognosis. The most common sites of extrahepatic metastasis are lung, regional lymph nodes, bone, adrenal glands, and peritoneum/omentum. Detection of such extrahepatic metastasis plays a vital role in the staging and treatment planning of HCC.

Case presentation: A 60-year-old man was presented to our centre with loss of apetite, generalised weakness, and weight loss. Abdominal examination revealed a firm lump in the right hypochondrium. CT findings revealed a large well-defined hypodense mass in almost entire right lobe of the liver. A well-defined oval, heterogeneously enhancing soft tissue mass lesions were also noted in both adrenal glands and psoas muscles. For histopathological diagnosis, percutaneous ultrasound-guided truecut biopsy was done from right lobe liver mass confirming well differentiated HCC.

Conclusions: In the present case report, we present an extremely rare and unique case of HCC with disseminated skeletal muscle metastasis with concomitant bilateral axillary lymph node metastasis. It is crucial for radiologists to detect such extrahepatic sites of metastasis initially at the time of diagnosis for accurate staging and treatment planning for a better prognosis.
\end{abstract}

Keywords: Hepatocellular carcinoma, Extrahepatic metastasis, Computed tomography (CT), Case report

\section{Background}

In 2020, liver cancer ranked sixth in incidence $(905,677$ new cases) and third in mortality (830,180 deaths) globally [1]. Hepatocellular carcinoma (HCC) is the predominant form and accounts for $90 \%$ of primary liver cancers. $\mathrm{HCC}$ is the most common primary liver malignancy with a high risk of metastasis. HCC hematogenous spread occurs via the lymphatic route or by direct invasion resulting in intrahepatic as well as extrahepatic metastasis [2].

The common sites of extrahepatic HCC metastasis with their frequencies are brain (2\%), peritoneum/omentum

\footnotetext{
*Correspondence: academics@manavatacancercentre.com

1 Department of Radio-Diagnosis, HCG Manavata Cancer Centre,

Nashik 422011, Maharashtra, India

Full list of author information is available at the end of the article
}

(11\%), adrenal glands (11\%), bone (28\%), regional lymph nodes $(53 \%)$, and lung $(55 \%)[3,4]$. Clinically, the presence of extrahepatic metastasis is considered as an advanced stage of HCC. Where such patients are not considered for curative treatment but treated with palliative intent as locoregional treatment or systemic chemotherapy. Due to its poor prognosis, the median survival was observed to be less than one year. Hence it is important to determine the presence of metastases at the time of initial HCC diagnosis for initiation of appropriate therapy, to determine the survival and follow-up $[5,6]$.

In the present case report, we described an unusual case of HCC presented with both common as well as uncommon sites of metastasis. Where rare multiple widespread skeletal muscle metastasis with concomitant bilateral adrenal and axillary lymph node metastasis was observed without common metastatic site lung. To 
the best of our knowledge, literature related to such rare occurrences has not been reported before.

\section{Case presentation}

A 60-year-old patient was presented to our centre with loss of apetite, generalised weakness, and weight loss. Patient was non alcoholic. Abdominal examination revealed a firm lump in the right hypochondrium. Routine blood tests, liver function tests, hepatitis viral marker, Serum Alpha Feto Protein (AFP, $1.6 \mathrm{ng} / \mathrm{ml}$ ), Carcinoembryonic Antigen (CEA, $2.2 \mathrm{ng} / \mathrm{ml}$ ), and ProstateSpecific Antigen (PSA, $0.5 \mathrm{ng} / \mathrm{ml}$ ) reports were all found to be within normal range.

Abdominal ultrasound was done, revealing a large heteroechoic mass in the right lobe and similar other lesions in the left lobe of the liver. No ascites were found and visualised portal vein shown normal colour flow.

To acquire additional information, a triple phase contrast enhanced CT (CECT) abdomen and pelvis was done. Where, a large well defined hypodense mass in almost entire right lobe of the liver (segment V/VI/VII/
VIII) measuring approximately $180 \times 30 \times 170 \mathrm{~mm}$ was seen with early arterial phase enhancement and early washout in the venous phase. Involvement of segmental portal vein was also observed. Whereas, rest of the portal vein branches and main portal vein has shown no intraluminal thrombus. Another morphologically similar lesion measuring $57 \times 46 \mathrm{~mm}$ was noted in segment II with early arterial phase enhancement and early washout in venous phase consistent with intrahepatic metastasis (Fig. 1).

A well-defined oval, heterogeneously enhancing soft tissue mass lesions were noted in both adrenal glands measuring approximately $60 \times 35 \mathrm{~mm}$ in the right adrenal gland and $45 \times 34 \mathrm{~mm}$ in the left adrenal gland suggesting bilateral adrenal metastasis (Fig. $2 \mathrm{C}$ and D). Multiple lytic lesions in vertebrae, bilateral ribs, and pelvic bones with largest in right iliac bone associated with soft tissue measuring $50 \times 36 \mathrm{~mm}$ was noted, suggesting bone metastasis (Fig. 2A, B, and D). Multiple soft tissue enhancing mass lesions were noted in both psoas muscles (Fig. 3A), in the anterior abdominal wall involving
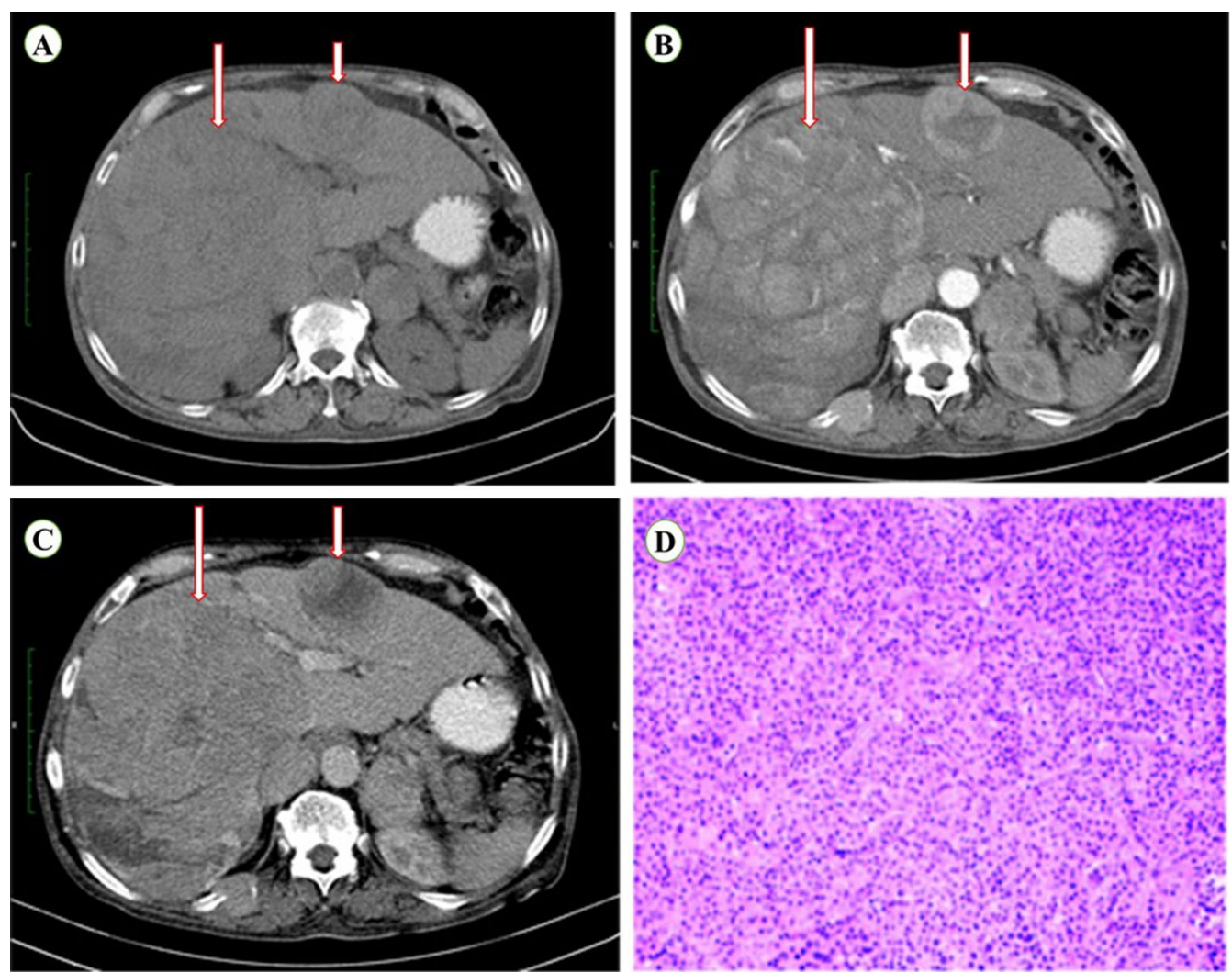

Fig. 1 Contrast axial CT images of the liver at various phases showing large well defined enhancing mass in the right lobe of the liver (large arrow) consistent with primary HCC and another enhancing small lesion in the left lobe of the liver (small arrow) suggesting intrahepatic metastases. A On a plain scan, both lesions appear hypodense, $\mathbf{B}$ arterial phase showing early arterial enhancement, $\mathbf{C}$ portal venous phase showing early washout. Imaging findings are consistent with HCC with intrahepatic metastasis, and $\mathbf{D}$ histopathology report of biopsy from right lobe liver mass showing well-differentiated HCC 

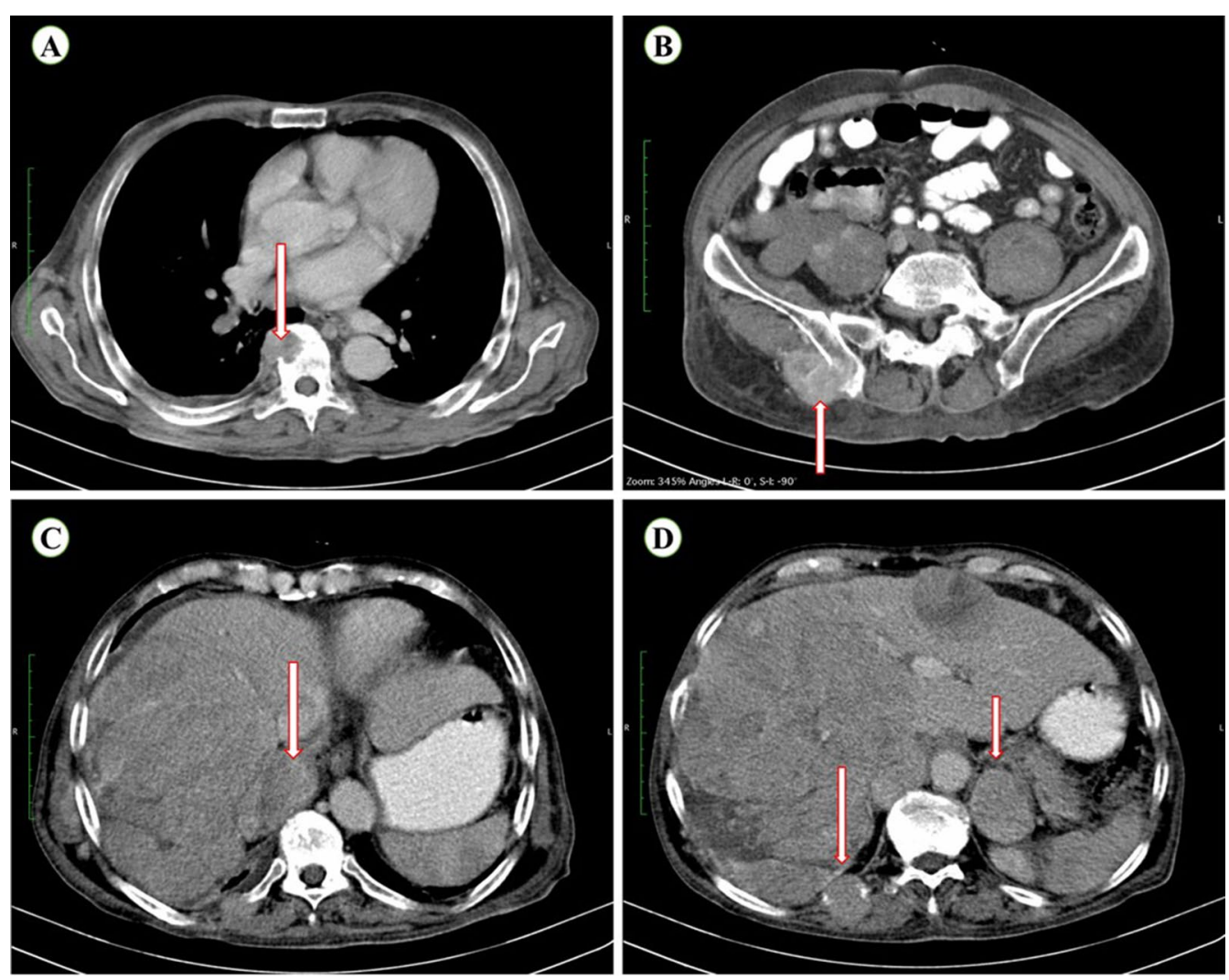

Fig. 2 Contrast axial CT images at various levels showing multiple lytic lesions $\mathbf{A}$ vertebrae, $\mathbf{B}$ right pelvic bone and $\mathbf{D}$ right side rib (large arrow) suggesting bone metastases. Also heterogeneously enhancing masses located at $\mathbf{C}$ right adrenal gland, and $\mathbf{D}$ left adrenal gland (small arrow) suggesting bilateral adrenal metastases

rectus abdominis muscles bilaterally measuring approximately $26 \times 23 \mathrm{~mm}$ in right rectus abdominis muscle and $60 \times 48 \mathrm{~mm}$ in left rectus abdominis muscle (Fig. 3B). Similar nodular soft tissue enhancing lesions were also noted in left gluteal muscles (Fig. 3C) and bilateral arm muscles (Fig. 3D) suggesting multiple muscle metastasis. However, multiple nodular soft tissue enhancing lesions within the muscles shown early arterial phase enhancement (Fig. 4A) and persistent enhancement in portal venous phase (4B), with delayed phase (Fig. 4C) unlike primary HCC showing washout in venous phase. To rule out lung metastasis, contrast enhanced CT thorax was done, showing no evidence of lung nodules (Fig. 5B). In addition, multiple enlarged bilateral axillary lymph nodes were noted, with the largest one measuring approximately $21 \times 17 \mathrm{~mm}$ in the left axilla suggesting metastatic lymph nodes (Fig. 5A). No significant mediastinal or hilar adenopathy or supraclavicular adenopathy was noted.

For histopathological diagnosis, percutaneous ultrasound-guided truecut biopsy was done from right lobe liver mass revealing well-differentiated HCC. Subsequently, percutaneous ultrasound-guided truecut biopsy from right rectus abdominis muscle lesion and fine needle aspiration cytology (FNAC) from left axillary lymph node were histopathologically analyzed confirming metastasis.

After initial diagnostic workup and confirmation of metastatic HCC, immunotherapy treatment was initiated using Injection Durvalumab (1500 mg, 9 cycles). Which was well tolerated by the patient with overall survival of 10 months.

\section{Discussion}

In patients with $\mathrm{HCC}$, early diagnosis and familiarity with its metastasis sites can profoundly impact the treatment options and prognosis. Correct risk stratification can help in avoiding unnecessary costs, delays in treatment, or disease related morbidities. The incidence of extrahepatic metastasis of $\mathrm{HCC}$ was reported to be about 15-17\% [7]. The most common sites of extrahepatic metastasis are lungs, adrenal glands, bones, and regional lymph nodes [4]. Metastasis to skeletal muscle and distant lymph nodes are uncommon. The factors associated with the risk of metastasis are alpha fetoprotein levels 

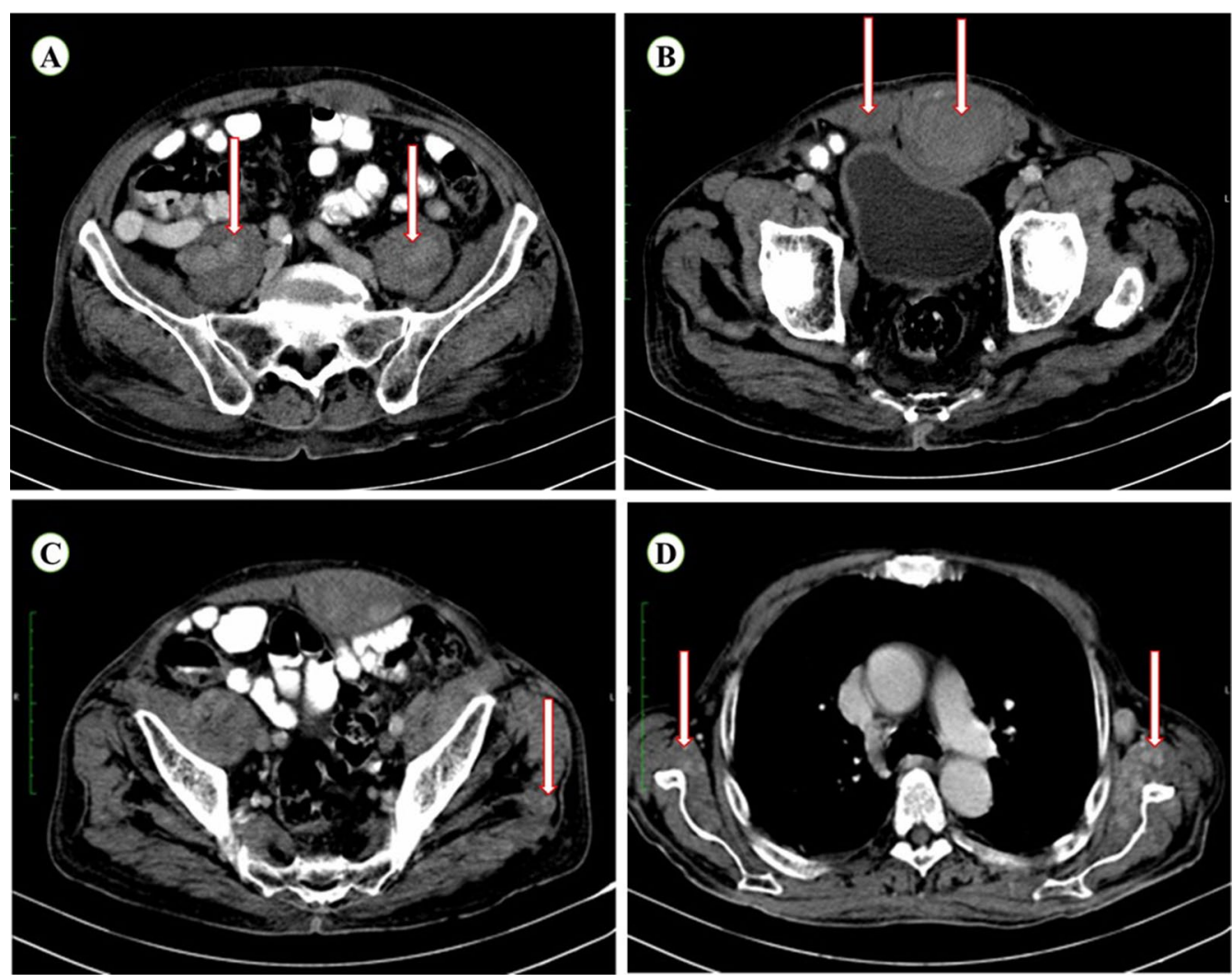

Fig. 3 Contrast axial CT images at various levels showing heterogeneously enhancing masses located at $\mathbf{A}$ bilateral psoas muscles, B bilateral rectus abdominis muscles, $\mathbf{C}$ left gluteal muscle, and $\mathbf{D}$ bilateral arm muscles suggesting widespread muscle metastasis

(AFP, $>400 \mu \mathrm{g} / \mathrm{ml}$ ), vascular invasion, index tumor size $(>5 \mathrm{~cm}$ ), and multifocal or infiltrative tumours [8].

Uchino et al. [9] conducted a study in 342 patients of HCC with extrahepatic metastasis. Where 28 patients were found to have extrahepatic metastasis on initial presentation and the remaining patients developed it during follow-up. The distribution of the metastasis among those patients was as followed-lung $(135,39.5 \%)$, lymph node $(117,34.2 \%)$, bone $(87,25.4 \%)$, adrenal (30, $8.8 \%)$, brain $(4,1.2 \%)$, spleen $(2,0.6 \%)$, and breast $(1$, $0.3 \%)$. The median survival after diagnosis of extrahepatic metastasis was reported to be 8.1 months. In a retrospective study by Katyal et al. [10], CT findings of 403 patients with HCC were reviewed and 148 patients were found to have extrahepatic metastasis. The most frequent site of metastasis in their patients was also found to be lungs (81, 55\%), abdominal lymph nodes $(60,41 \%)$, and bones (41, 28\%). Both Uchino et al. [9] and Katyal et al. [10] reported adrenal gland metastasis in $8.8 \%$ and $11 \%$ of patients, respectively. The adrenal gland metastasis was considered as the fourth most frequent site of metastasis after the lungs, lymph nodes, and bone. In our patient also multiple lytic bone metastasis and bilateral adrenal metastasis were also seen. However, the most common site of metastasis-lung was found to be normal without any sign of metastasis.

Other forms of metastasis include muscle and skin metastasis. Which in generally may occur via needle seedling post biopsy or by the surgery itself. But the risk of metastasis through this process was found to be as low as $2.7 \%$. However, this is different from muscle metastasis due to hematogenous spread. Intramuscular HCC is another rare metastasis, where very few reports in the literature have reported its presence in para vertebral muscle [7], right gluteal muscle [11], psoas muscle [12], intercostal muscle [13], left iliacus [14], multiple soft tissues [15], pectineal muscle [16], and chest wall [16]. It was also observed that even after curative surgery (hepatic resection/transplantation) or loco-regional treatment (TACE-TransArterial ChemoEmbolisation or TARE-TransArterial Radioembolisation) [12, 16, 17]. For primary HCC, high chances of developing extra hepatic metastasis was reported during the follow-up period.

In the present case report, our patient has also shown signs of muscle metastasis. However, its extent was 

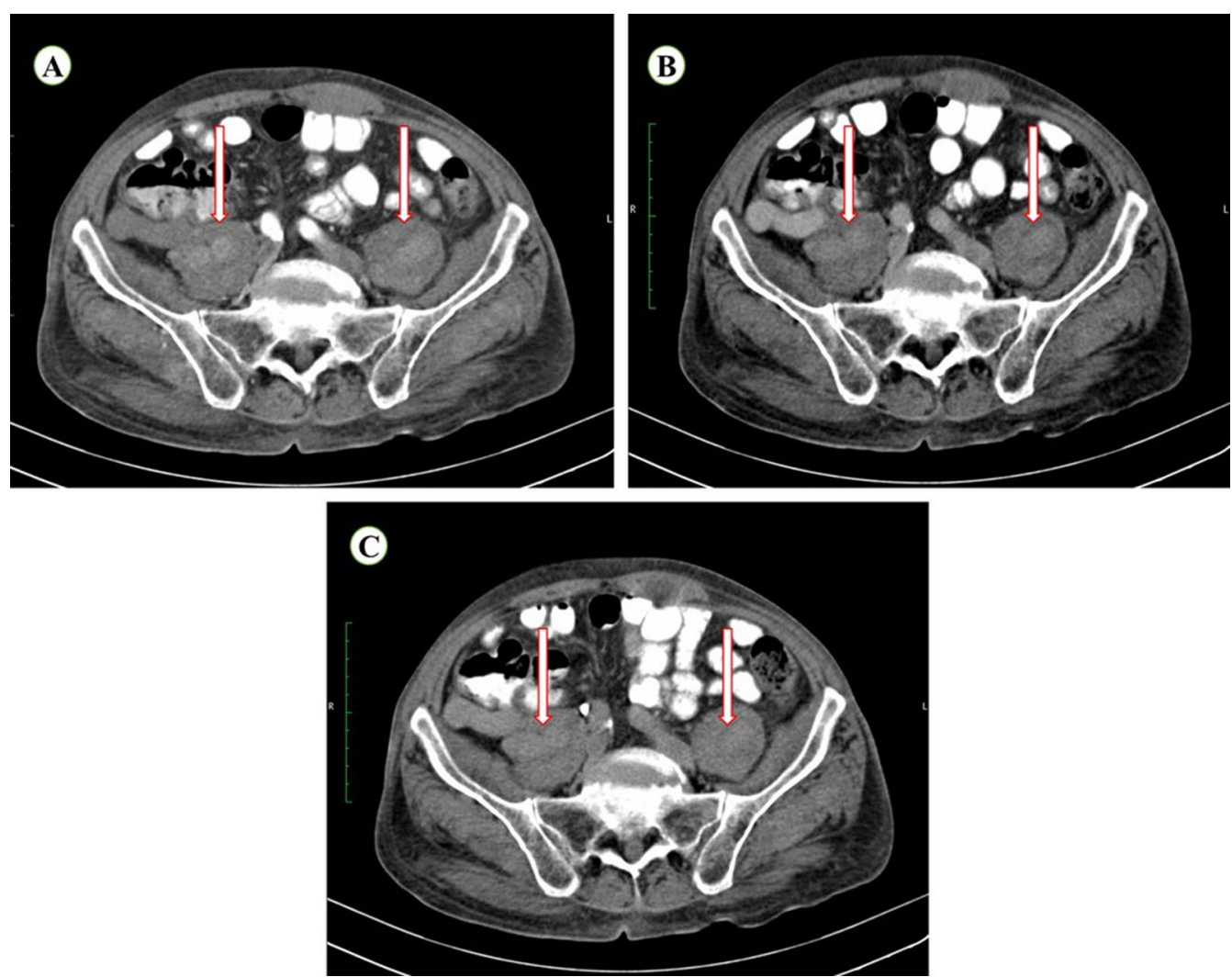

Fig. 4 Contrast axial CT images focused on the lesions at psoas muscles in different acquisition phases, $\mathbf{A}$ marked enhancement during the arterial phase, $\mathbf{B}$ portal venous phase, and $\mathbf{C}$ delayed phase showing persistent enhancement and no washout
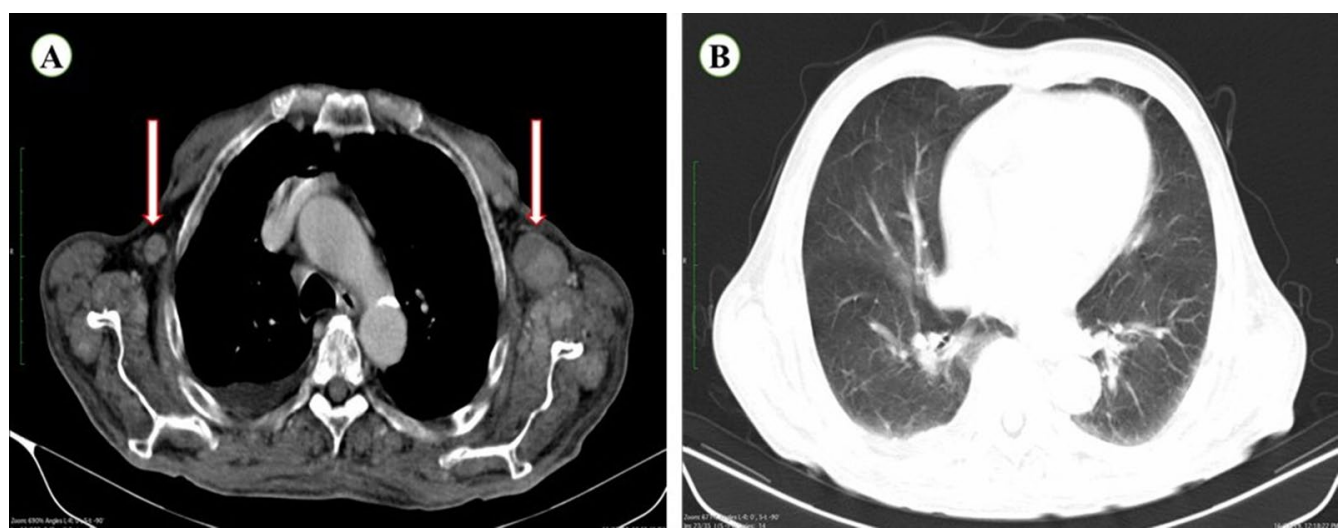

Fig. 5 Contrast axial CT image of thorax showing $\mathbf{A}$ enhancing enlarged bilateral axillary lymph nodes suggesting axillary lymph node metastasis, and $\mathbf{B}$ normal lungs with no evidence of lung metastases

completely different compared to earlier literature. Where a widespread multiple muscle metastasis was found in the abdomen, paravertebral, and arms. The muscle metastasis on triphasic CT scan has also shown an early arterial enhancement without washout in portal venous or venous phase as seen in HCC.

Another common site of metastasis is regional abdominal lymph nodes. However, distant lymph node 
metastasis can also present with the most common site as mediastinal lymph nodes [16]. In our patient, a very rare enlarged bilateral axillary lymph nodes were detected. Only one case report was found in the literature involving the axillary lymph nodes by metastases from HCC [16]. Possible routes for such metastatic spread to axillary lymph nodes might be through the primary tumor involving the upper part of the right liver lobe (under the bare area of the liver) where it spreads via lymphatic vessels to reach parasternal or mediastinal lymph nodes to the axillary lymph nodes. Another alternate possible route for such metastasis may be from the portal venous system to the umbilical region [18]. Whereas in our patient, bilateral axillary lymph node metastasis was seen without mediastinal lymph node involvement itself.

Thus the present case report has the peculiarities as mentioned:

1. It described common sites of metastasis as bone and adrenal glands other than lung, which is the first most frequent site of metastasis.

2. Uncommon sites of metastasis to muscles with widespread multiple muscle involvement, which were not described earlier.

3. Uncommon site of metastasis such as axillary lymph nodes. Which was bilateral and not reported yet in literature.

\section{Conclusions}

We report the first case of HCC with bilateral axillary lymph node involvement with widespread muscle metastasis. Though this is a single case, it can raise interest in clinicians performing imaging by helping in to focus and identify the uncommon metastasis sites of HCC for patient's overall well-being and timely treatment. It is crucial for radiologists to detect such extrahepatic sites of metastasis initially at the time of diagnosis for accurate staging and treatment planning, as well as in follow up cases (as post resection or post locoregional therapy) to evaluate for recurrence.

\section{Abbreviations}

HCC: Hepatocellular carcinoma; CT: Computed tomography; AFP: Alpha Feto Protein; CEA: Carcinoembryonic Antigen; PSA: Prostate-Specific Antigen.

\section{Acknowledgements}

The authors would like to thank Dr. Yasam Venkata Ramesh from HCG Manavata cancer centre, Centre for difficult cancers (CDC), Nashik, India, for his medical writing assistance.

\section{Authors' contributions}

Concept—M.T.; Design-M.T., Y.V.R.; Supervision-M.T., R.N.; Materials—M.T., R.N.; Data Collection and/or Processing-M.T., Y.V.R.; Analysis and/or Interpretation-M.T., Y.V.R., R.N.; Literature Search-M.T., Y.V.R.; Writing Manuscript-Y.V.R.; Critical Review-M.T., Y.V.R. All the authors have participated sufficiently in contributing to the content of'Hepatocellular carcinoma with uncommon sites of metastasis: A rare case report' and have read and approved the manuscript. All authors read and approved the final manuscript.

Funding

We declare that our work is not funded by any institution, organization, or government, and we have no financial support.

\section{Availability of data and materials}

The data used during the current study are available from the corresponding author on reasonable request.

\section{Declarations}

\section{Ethical approval and consent to participate}

Ethical approval is not obtained, since it is a retrospective case report study. Consent was obtained from the patient for publication of anonymized images.

\section{Consent for publication}

A written consent was obtained from the patient for publication of data and anonymized images.

\section{Competing interests}

The authors declare that they have no competing interests.

\section{Author details}

${ }^{1}$ Department of Radio-Diagnosis, HCG Manavata Cancer Centre,

Nashik 422011, Maharashtra, India. ${ }^{2}$ Department of Academics, HCG Manavata Cancer Centre, Nashik 422011, Maharashtra, India. ${ }^{3}$ Department of Surgical

Oncology, HCG Manavata Cancer Centre, Nashik 422011, Maharashtra, India.

Received: 6 July 2021 Accepted: 15 September 2021

Published online: 21 September 2021

\section{References}

1. 2020 G. Liver [Internet]. [cited 2021 Jun 2]. https:/gco.iarc.fr/today/data/ factsheets/cancers/11-Liver-fact-sheet.pdf

2. Sneag DB, Krajewski K, Giardino A, O'Regan KN, Shinagare AB, Jagannathan JP et al (2011) Extrahepatic spread of hepatocellular carcinoma: spectrum of imaging findings. Am J Roentgenol 197(4):W658-W664

3. Becker AK, Tso DK, Harris AC, Malfair D, Chang SD (2014) Extrahepatic metastases of hepatocellular carcinoma: a spectrum of imaging findings. Can Assoc Radiol J 65:60-66

4. Natsuizaka M, Omura T, Akaike T, Kuwata Y, Yamazaki K, Sato T et al (2005) Clinical features of hepatocellular carcinoma with extrahepatic metastases. J Gastroenterol Hepatol 20:1781-1787

5. Ishii H, Furuse J, Kinoshita T, Konishi M, Nakagohri T, Takahashi S et al (2004) Extrahepatic spread from hepatocellular carcinoma: who are candidates for aggressive anti-cancer treatment? Jpn J Clin Oncol 34:733-739

6. Greten TF, Papendorf F, Bleck JS, KirchhoffT, Wohlberedt T, Kubicka S et al (2005) Survival rate in patients with hepatocellular carcinoma: a retrospective analysis of 389 patients. $\mathrm{Br} J$ Cancer 92:1862

7. Takahashi K, Putchakayala KG, Safwan M, Kim DY (2017) Extrahepatic metastasis of hepatocellular carcinoma to the paravertebral muscle: a case report. World J Hepatol 9:973-978

8. Yokoo T, Patel AD, Lev-Cohain N, Singal AG, Yopp AC, Pedrosa I (2017) Extrahepatic metastasis risk of hepatocellular carcinoma based on a-fetoprotein and tumor staging parameters at cross-sectional imaging. Cancer Manag Res 9:503-511

9. Uchino K, Tateishi R, Shiina S, Kanda M, Masuzaki R, Kondo Y et al (2011) Hepatocellular carcinoma with extrahepatic metastasis: clinical features and prognostic factors. Cancer 117:4475-4483

10. Katyal S, Oliver JH, Peterson MS, Ferris JV, Carr BS, Baron RL (2000) Extrahepatic metastases of hepatocellular carcinoma. Radiology 216:698-703

11. Young C, Munk PL (2007) Hepatocellular carcinoma presenting as musculoskeletal metastases: a report of two cases. Eur J Radiol Extra 62:25-29 
12. Wu MH, Wu YM, Lee PH (2006) The psoas muscle as an unusual site for metastasis of hepatocellular carcinoma: report of a case. Surg Today 36:280-282

13. Furumoto K, Miura K, Nagashima D, Kojima H, Mori T, Ito D et al (2012) Solitary metastasis to the intercostal muscle from hepatocellular carcinoma: a case report. Int J Surg Case Rep 3:322-326

14. Subramaniam N, Hiremath B, Pujar A (2013) Metastasis of diffuse hepatocellular carcinoma to an extremely unusual site. BMJ Case Rep 2013:bcr2013200437

15. Ananthkumar S (2017) Hepatocellular carcinoma with multiple soft tissue metastasis - a rare case report

16. Sirigu D, Loi L, Mura R, Migaleddu V, Campisi G (2009) Muscle metastasis from hepatocellular carcinoma in a patient treated with TACE. J Ultrasound $12: 45-47$
17. Onen A, Sanli A, Karacam V, Karapolat S, Gokcen B, Acikel U (2008) Chestwall metastasis in a patient who underwent liver transplantation due to hepatocellular carcinoma. Hear Lung Circ 17:156-158

18. Alison MR, Leiman G, Kew MC (2000) Metastasis in an axillary lymph node in hepatocellular carcinoma: a case report. World J Gastroenterol 6:770-772

\section{Publisher's Note}

Springer Nature remains neutral with regard to jurisdictional claims in published maps and institutional affiliations.

\section{Submit your manuscript to a SpringerOpen ${ }^{\circ}$ journal and benefit from:}

- Convenient online submission

- Rigorous peer review

- Open access: articles freely available online

- High visibility within the field

- Retaining the copyright to your article

Submit your next manuscript at $\boldsymbol{\nabla}$ springeropen.com 